\title{
Candidiasis invasoras en el paciente crítico adulto
}

\author{
Eduardo Tobar A., Francisco Silva O., Roberto Olivares C., Pablo Gaete G. y Mario Luppi N.
}

\section{Invasive candidiasis in critically ill adult patient}

Invasive infections by Candida strains are a relevant pathology in critically ill patients. Candida should be considered where a high risk of infection is present for a critical early diagnosis. Despite the incorporation of new drugs in the therapeutic armamentarium over the last decade, mortality remains high. The key in improving clinical outcomes of these patients are the use of early effective therapies that offer coverage against different strains of Candida: C. albicans and non-albicans. Recent international guidelines suggest empiric therapy with echinocandins in suspected invasive candidiasis in this patient population. This group of drugs adequately documented clinical efficacy and safe use in these patients. The emergence of new echinocandins could improve access to these drugs by reducing their cost.

Key words: Mycoses Candida spp, fungal infection, critical illnesses, echinocandins.

Palabras clave: Micosis cándida, infección fúngica, paciente crítico, equinocandinas.

\section{Introducción}

a candidiasis invasora $(\mathrm{CI})$ es una patología importante en la población de pacientes críticos, dada su frecuencia, elevada mortalidad y asociación a un incremento en los costos y estadía hospitalaria. Ésta se define como el aislamiento de alguna especie de Candida, en hemocultivos (candidemia) y/o en algún otro sitio estéril infectado por este patógeno. El cuadro clínico se manifiesta como un complejo conjunto de síntomas y signos, sin tener en general, elementos clínicos que lo hagan fácilmente distinguible de otras infecciones. Para su adecuado diagnóstico se requiere de un elevado nivel de sospecha y la adecuada valoración de factores de riesgo, síntomas y signos clínicos, así como de la presencia de colonización por cándida en otros sitios.

Candida spp, corresponde a un grupo de hongos, del cual en la actualidad se han identificado más de 100 especies con el potencial de afectar al ser humano. Candida albicans, el más tradicional representante de esta familia $\mathrm{u}$ otras especies de Candida, forma parte normal de la microbiota humana. Sus manifestaciones patológicas más frecuentes son las infecciones superficiales, por ejemplo vulvovaginitis o candidiasis oral. Diferentes especies de Candida pueden producir también compromiso específico de algunos órganos en pacientes que, en general, poseen enfermedades crónicas que los sitúan en condición de inmunodeficiencia (p. ej.: candidiasis esofágica). En una proporción menor de pacientes, en particular aquellos que se encuentran hospitalizados, bajo terapia con antimicrobianos de amplio espectro, que se encuentran invadidos con algún dispositivo biomédico (catéter central, tubo oro-traqueal, entre otros) y/o con otros factores de riesgo, se presentan infecciones fúngicas invasoras.

Para esta última condición se describe una mortalidad cruda de 40 a $70 \%$, y una mortalidad atribuible de 20 a $40 \%$. Además del impacto en mortalidad, su presencia se asocia a un significativo incremento en los costos de la atención y en la duración de la estadía hospitalaria. Lamentablemente, no existe a la fecha un test diagnóstico precoz para esta entidad, quedando su adecuado diagnóstico al buen juicio clínico del equipo médico tratante, quienes deben sopesar la presencia de factores de riesgo clínicos, la presencia de colonización por cándida y la condición clínica del paciente para definir el inicio de terapia antifúngica empírica ante la sospecha de CI. Esta información se complementa con el aislamiento de Candida sp en el cultivo de sitios estériles, aunque existe una limitación en la sensibilidad y en el tiempo de respuesta de los métodos para diagnóstico microbiológico habitual.

El objetivo de la presente revisión es describir la epidemiología de la CI, los mecanismos que la originan, el cuadro clínico y las terapias antifúngicas recomendadas. Un énfasis particular se da a la discusión acerca del empleo de equinocandinas planteado en las recientes recomendaciones de la IDSA (Sociedad Americana de Infectología $)^{1}$.

\author{
Hospital Clínico Universidad \\ de Chile, Santiago. \\ Departamento de Medicina \\ Unidad de Paciente Crítico \\ Medicina Intensiva (ETA). \\ Sección Infectología (ROC, PGG, \\ MLN). \\ Laboratorio de Microbiología (FSO) \\ Declaración de conflictos interés: \\ Los Dres. Tobar y Olivares han \\ sido patrocinado por MSD en \\ su participación en congresos \\ científicos, además son \\ investigadores de Pfizer en estudio \\ clínico A8851015. Los Dres. Gaete \\ y Luppi han sido patrocinados \\ por MSD en su participación \\ en congresos científicos, y \\ participado como conferencistas \\ en conferencias patrocinadas por \\ MSD y Pfizer. \\ El Dr. Silva declara no tener \\ conflictos de interés.
}

Recibido: 1 de marzo 2010 Aceptado: 28 de septiembre 2010

Correspondencia a: Eduardo Tobar Almonacid etobar@redclinicauchile.cl 


\section{Epidemiología}

Recientes reportes sitúan a la candidemia entre el $4^{\circ}$ y $5^{\circ}$ lugar de los aislados positivos de hemocultivos en pacientes hospitalizados, con un total de 8 a $15 \%$ de hemocultivos positivos ${ }^{2,3}$. En Latinoamérica se ha descrito una incidencia elevada, de hasta 2,49 casos / 1.000 admisiones, lo que corresponde a 0,37 casos por 1.000 pacientes-día ${ }^{4,4 a}$. No existen a la fecha estudios chilenos de incidencia.

La mortalidad cruda descrita para esta entidad varía entre 35 y $75 \%$. Esta variación depende, entre otros factores, de las características del grupo de pacientes estudiado (co-morbilidad, gravedad previa a la aparición de candidemia) y del país donde se efectuó el análisis ${ }^{5-7}$.

Existe controversia en la literatura médica respecto a cuánto de la elevada mortalidad, corresponde a "mortalidad atribuible", con estudios que la sitúan en un amplio margen de 5 a $70 \%{ }^{6,8}$. Muy probablemente, entre 30 y $50 \%$ de los fallecidos sean directamente atribuibles a la infección fúngica, ello sin considerar el reconocido subdiagnóstico de esta condición, como lo reflejan estudios realizados post mortem.

Otro dato relevante y en cual si disponemos de información nacional, ha sido el progresivo incremento en la importancia relativa de las cándidas no albicans, las cuales han pasado como grupo a representar alrededor de 50\% de los aislados de Candida en hemocultivo ${ }^{9,10}$.

Respecto al comportamiento epidemiológico de la CI en la unidad de cuidados intensivos (UCI), existen escasos reportes en la literatura científica que analicen la incidencia en esta población de pacientes en particular. Sí es claro en los estudios epidemiológicos efectuados, que entre 45 y $70 \%$ de los pacientes con candidemia provienen de la UCI. Asimismo, se ha documentado que la incidencia de candidemia en UCI es de alrededor de 7 veces superior a la observada en pacientes de sala ${ }^{11,12}$.

Dentro de los estudios que abarcan exclusivamente pacientes admitidos a UCI, se halla el de Bassetti y cols. Éste revela un incremento en la incidencia de candidemia en 5 años de observación, desde 1,25 a 3,06 episodios $/ 10.000$ pacientes días-año, así como un incremento en la proporción de cándida no albicans ${ }^{13}$.

Otro hecho relevante del punto de vista epidemiológico es que recientemente se ha descrito un incremento en los aislados en sangre de Candida spp en infecciones adquiridas en la comunidad ${ }^{14,15}$. Una proporción elevada de estos episodios, sin embargo, se ubican más bien bajo el concepto de infecciones asociadas a los cuidados de la salud, al tratarse de pacientes institucionalizados, portadores de catéter urinario permanente, haber egresado recientemente del hospital o estar en presencia de algún otro factor de riesgo. En la actualidad, todavía debemos considerar que la mayor parte de las infecciones fúngicas invasoras por cándida se presenta en la población de pacientes críticamente enfermos, como una complicación endógena tardía aparecida durante su estadía en UCI.

Así, el individuo se coloniza por Candida spp en presencia de factores de riesgo y dispositivos invasores y, en su contexto de déficit inmunitario asociado a la enfermedad grave, se manifiesta como enfermedad sistémica.

Aunque este es el mecanismo predominante para las infecciones fúngicas invasoras por Candida spp, se ha descrito la presencia de infección exógena, generando brotes nosocomiales de candidemia, en relación a la trasgresión de recomendaciones en la prevención de infecciones asociadas a los cuidados de salud. Ello fue inicialmente reportado en población neonatal ${ }^{16}$, pero a la fecha hay reportes de brotes causados por diversas especies de cándida en pacientes de diversas dependencias como unidades hematológicas y unidades de cuidados intensivo de adultos ${ }^{17}$.

\section{Manifestaciones clínicas}

El diagnóstico presuntivo de $\mathrm{CI}$ es fundamentalmente clínico y se basa en la aparición de signos y/o síntomas de infección, en un paciente con factores de riesgo y/o colonización documentada por Candida spp.

Dentro de los síntomas y signos se encuentran la presencia de fiebre, taquicardia e hipotensión arterial; lamentablemente, estos hallazgos son inespecíficos presentándose también en otras complicaciones infecciosas de pacientes admitidos a UCI. Las candidemias se presentan con invasión tisular en menos de $3 \%$ de los casos y las lesiones dérmicas secundarias no llegan a 10\% de los mismos. Por ello no se recomienda la búsqueda dirigida de compromiso visceral, a excepción de los pacientes que desarrollan candidemias persistentes o evidencia de falla a tratamiento ${ }^{18}$. Pese a lo anterior, el examen de fondo de ojo se debe realizar rutinariamente a estos pacientes, dada su fácil disponibilidad y bajo costo.

En la perspectiva del médico intensivista, el paciente se manifiesta habitualmente como un nuevo cuadro de sepsis grave, con la presencia de un síndrome de respuesta inflamatoria sistémica (SRIS) de intensidad variable. Ello se presenta, por lo general, en la segunda o tercera semana de estadía en la unidad de paciente crítico. Ha sido documentado que la candidemia puede manifestarse de forma aislada como shock séptico, lo que se asocia a una elevada presencia de falla multiorgánica y mortalidad ${ }^{19}$.

Como antes se ha señalado, ninguna de las manifestaciones clínicas de CI es específica ni está presente en más de 70 a $80 \%$ de los casos, existiendo una proporción de pacientes en los que el cuadro clínico es poco manifiesto e incluso sólo se documenta en la necropsia ${ }^{20}$. Por ello, en presencia de un cuadro de "nueva fiebre en el paciente crítico" y/o de reaparición de disfunción cardiovascular, es necesario considerar la presencia de infección invasora 
por cándida. La aproximación tradicional que se realiza en pacientes críticos en quienes se sospecha un segundo evento infeccioso es la evaluación sistemática de las complicaciones infecciosas más frecuentes como son la neumonía asociada a ventilación mecánica, la infección del torrente sanguíneo asociada a catéter venoso central, la infección del tracto urinario asociada a catéter urinario y la búsqueda de complicaciones locales en el paciente quirúrgico. Esta aproximación debería incorporar siempre una evaluación del riesgo de presentar una CI como recomiendan recientes guías del enfrentamiento del paciente febril en $\mathrm{UCI}^{21}$, ya que uno de los errores frecuentes que se cometen en este escenario es la no sospecha clínica de esta condición, haciéndose el diagnóstico en forma tardía.

Ante la ausencia de un cuadro clínico específico se ha sugerido que para favorecer el diagnóstico clínico precoz de esta condición sería necesario el reconocimiento de los factores de riesgo clínicos y de la presencia de colonización por Candida spp. Las especies de Candida son parte frecuente de la microbiota comensal de individuos sanos pero, en condiciones de inmunocompromiso, exposición a antimicrobianos e invasión por dispositivos biomédicos, este hallazgo puede traducir una infección sistémica. Los pacientes críticos son una población en los que se concentran estos factores de riesgo, motivo que explica el elevado riesgo de desarrollar CI en este grupo, en comparación con pacientes de menor complejidad. Los principales factores de riesgo para la aparición de infección invasora por Candida mencionados en la literatura médica se señalan en la Tabla 1.

\section{Diagnóstico}

Además del reconocimiento de la presencia de factores de riesgo clínicos, se ha documentado que la colonización por Candida spp en piel, mucosas o vía aérea, es un evento que habitualmente precede a la infección sistémica ${ }^{22}$. En virtud de ello, se han tratado de desarrollar sistemas de puntuación que faciliten el diagnóstico precoz y la decisión del inicio empírico de antifúngicos. Hace ya 15 años,

\section{Tabla 1. Factores de riesgo para el desarrollo de} candidemia $y / 0$ candidiasis invasora ${ }^{10,45-47}$

\begin{tabular}{l} 
Estadía en UCI \\
Uso de catéter venoso central \\
Insuficiencia renal \\
Cirugía abdominal \\
Pancreatitis aguda \\
Neutropenia \\
Uso de antimicrobianos de amplio espectro \\
Nutrición parenteral central \\
\hline
\end{tabular}

Pittety cols desarrollaron un sistema de puntuación basado en la presencia de colonización por cándida ${ }^{23}$. Recientemente, el grupo español colaborativo en la investigación de infecciones fúngicas desarrolló el denominado "Puntaje Cándida", el cual podría tener un rol en la decisión de no iniciar terapia empírica, al documentarse un elevado valor predictor negativo de este sistema de puntuación ${ }^{24,25}$. Ello se traduce que en presencia de menos de tres puntos en este sistema es muy improbable que un paciente presente esta condición (Tabla 2). Es necesario considerar que en todos los sistemas de puntuación estudiados para estratificar el riesgo de CI, la búsqueda de colonización por cándida se efectúa mediante vigilancia microbiológica sistemática de los sitios colonizados, lo que incorpora sitios no evaluados habitualmente por el médico clínico. En este score en particular se evalúan al ingreso, y luego cada semana hasta el egreso, los siguientes sitios o muestras: aspirado traqueal, exudado oro-faríngeo, aspirado gástrico y orina. Pueden considerarse además muestras tales como deposiciones, exudados de heridas, drenajes y algunos más evidentes como sangre y líneas endovasculares.

El Puntaje Cándida, solo o en conjunto con otros biomarcadores, parece en camino a convertirse en un apoyo real para el diagnóstico de CI; sin embargo, es necesario destacar que requiere la búsqueda semanal de sitios colonizados como aspirado traqueal, hisopado rectal, orina, piel, aspirado gástrico y sangre. La costo-efectividad de esta estrategia en escenarios con baja incidencia de CI y de bajo empleo de antifúngicos aún no está definida.

Parte de las dificultades diagnósticas de esta condición se debe además a que a la fecha no hay técnicas de laboratorio rápido que permitan guiar el inicio de la terapia antifúngica empírica. Se encuentran en desarrollo técnicas de medicina molecular (reacción de polimerasa en cadena, RPC), de medición de algunas moléculas producidas por Candida spp, como la detección de anticuerpo y antígeno manana, $\alpha$ y $\beta$ oligomanosas, anticuerpo a tubo germinativo en C. albicans (CAGTA) ${ }^{26,28}$. También están en desarrollo en el laboratorio de microbiología técnicas que nos permitan facilitar el diagnóstico de infección sistémica por cándida a través de la detección del componente de la pared fúngica 1,3 $\beta$-D-glucano. Estas técnicas, así como la RPC cuentan a la fecha con datos alentadores, con estudios

\begin{tabular}{|l|}
\hline \multicolumn{1}{|c|}{ Tabla 2. Puntaje Cándida ${ }^{24-25}$} \\
\hline Cirugía al ingreso a UCl \\
Nutrición parenteral central (NPC) \\
Sepsis grave \\
Colonización multifocal por Candida sp \\
\hline $\begin{array}{l}\text { Puntaje Cándida }=1 \times(\mathrm{NPC})+1 \times \text { (cirugía) }+1 \times \text { (colonización } \\
\text { multifocal) }+2 \times \text { sepsis grave. Las variables codificadas como } 0: \\
\text { ausente; } 1 \text { : presente. }\end{array}$ \\
\hline
\end{tabular}


que muestran una elevada sensibilidad y especificidad para el diagnóstico de CI.

Pese a estos avances, aún se espera un mayor desarrollo y validación de estas nuevas técnicas, por lo que el grado de sospecha clínica y la estratificación de riesgo según sea la presencia de colonización por cándida y/o factores de riesgo se mantienen como el eje diagnóstico.

Los hemocultivos son fundamentales en el diagnóstico de la CI. Para optimizar su rendimiento deben obtenerse dos o tres muestras mediante punción de vía periférica, en cantidad superior a $20 \mathrm{ml}$, y ser procesadas por métodos automatizados. Independientemente del número de hemocultivos obtenidos, el valor predictor de candidemia de sólo un cultivo positivo es alto y requiere tratamiento. $\mathrm{Si}$ el hemocultivo está asociado a presencia de catéter venoso central, éste debe ser retirado. En pacientes neutropénicos hay clara evidencia de una mayor mortalidad si los dispositivos endovasculares se preservan y, en el paciente crítico, el manejo no debiese ser distinto en este aspecto.

Debemos reconocer eso sí, la baja sensibilidad de los métodos microbiológicos (hemocultivo automatizado) que alcanza alrededor de 50 a $60 \%$. Además debemos considerar el tiempo habitual de demora entre la toma de la muestra y el aislamiento e identificación de la especie de Candida, el cual con frecuencia tarda 72 a 96 horas.

Si se configura el escenario de sospecha clínica de infección fúngica invasora por Candida spp, lo recomendado entonces es la toma de cultivos (sangre y de otros sitios bajo la sospecha clínica) e inicio de terapia antifúngica.

En algunos casos, además de evidenciar mediante el cultivo la presencia de Candida spp, se hace necesario conocer la susceptibilidad a los diferentes antifúngicos, en especial a fluconazol, dada la emergencia de cepas no susceptibles de $C$. albicans y de algunas otras especies como Candida glabrata, C. tropicalis y C. parapsilosis ${ }^{29}$. A esto se suma el reporte de cepas resistentes a equinocandinas o con concentración inhibitoria mínima (CIM) elevada para anfotericina $\mathrm{B}$.

En la actualidad existen dos recomendaciones internacionales para determinar la susceptibilidad antifúngica frente a especies de Candida, la del Clinical Laboratory Standards Institute (CLSI) y del European Committee on Antimicrobial Susceptibility Testing (EUCAST), los cuales difieren en la metodología y puntos de corte, por lo que los resultados utilizando uno u otro método no son extrapolables entre sí.

No existe un claro acuerdo acerca de en qué casos hay que realizar estudio de susceptibilidad pero, en nuestro parecer, sería recomendable en las siguientes situaciones:

- Aislados de Candida spp provenientes de sitios estériles, en especial cuando se trate de Candida no albicans. No es necesario contar con susceptibilidad a fluconazol en aislados de C. krusei ya que es una especie intrínsecamente resistente.
- En casos de pacientes con infecciones que no responden a terapia o que presentan infección por Candida spp mientras se encuentran en profilaxis o tratamiento antifúngico.

- En pacientes con infecciones recurrentes por Candida spp o con antecedentes de tratamientos prolongados con azólicos.

\section{Terapia}

Para el adecuado tratamiento de la CI es necesaria una terapia antifúngica óptima, lo que implica al menos cuatro conceptos:

- Adecuada cobertura de la especie de Candida identificada (eficacia en la erradicación microbiológica).

- Un perfil de seguridad adecuado en un grupo de pacientes que frecuentemente tiene alteraciones en la función cardiovascular, renal y/o hepática.

- El momento adecuado para el inicio de la terapia antifúngica.

- El costo de las alternativas disponibles.

En conjunto con la decisión del antifúngico a emplear, sus dosis y tiempos de empleo, es necesario destacar algunos aspectos generales en el manejo de la sospecha de CI. En primer lugar, se recomienda el retiro de catéteres centrales en pacientes con candidemia, debido a la posible colonización de ellos. Ello es posible la mayor parte de las veces; sin embargo, en algunos pacientes sin opción de otro acceso, podrían tomarse cultivos pareados de los lúmenes y certificar la ausencia de colonización por cándida. En los pacientes quirúrgicos, siempre realizar búsqueda de complicación intra-abdominal, y proceder al drenaje quirúrgico o percutáneo de las colecciones documentadas. Finalmente, aunque la nutrición parenteral central ha sido descrita como factor de riesgo de candidemia, ello parece deberse más a que su uso es un marcador de gravedad. De todas formas, y como en otras condiciones de los pacientes críticos, debiera privilegiarse el soporte nutricional por vía enteral en la medida que las condiciones del paciente lo permitan.

Una consideración inicial a efectuar en el análisis del empleo de antifúngicos en pacientes críticos es la indicación bajo la cual se efectuó.

Esta puede ser clasificada como el empleo de terapia precoz o anticipatoria (pre-emptive therapy), uso empírico (en pacientes con sospecha clínica de CI) y uso documentado (en pacientes con un cultivo positivo para Candida spp en un sitio estéril).

Existen ensayos clínicos que solventan el empleo de fluconazol en pacientes críticos quirúrgicos con riesgo elevado de desarrollar CI. En estos pacientes, el empleo de fluconazol bajo el concepto de terapia preventiva 
reduce el riesgo de desarrollar candidemia, aunque el impacto en mortalidad no es claro ${ }^{30,31}$. Sin embargo, los estudios clínicos han sido confusos en las definiciones empleadas, confundiéndose en ocasiones la terapia precoz con empírica y siendo difícil obtener conclusiones válidas. Por otra parte, el empleo indiscriminado de fluconazol en este escenario, podría propiciar un incremento en la proporción de Candida spp resistentes a este fármaco y su empleo tiene la limitación del incremento de costos en la atención y del riesgo -aunque bajo-de la aparición de efectos adversos a fluconazol.

En nuestra opinión, no existe suficiente evidencia para su uso preventivo en la población de pacientes críticos, justificándose el empleo juicioso de terapia empírica, en particular, en pacientes críticos quirúrgicos, en los que aparecen elementos de inflamación y/o de infección activos y concentran factores de riesgo para el desarrollo de CI.

Diversas familias de antifúngicos están disponibles en el mercado. Sus características generales para el tratamiento de esta condición se observan en la Tabla 3. Las dosis y plazos recomendados de tratamiento para cada uno de los antifúngicos, se presentan en la Tabla 4.

Respecto a los antifúngicos disponibles, es importante destacar algunas ventajas y desventajas comparativas que poseen.

Fluconazol. Es en la actualidad el fármaco más empleado para el tratamiento de esta infección, tanto en el inicio de terapia empírica, como ante la confirmación de un aislado de cándida susceptible. Es relativamente barato y accesible; no obstante, el intenso empleo que se ha hecho de él durante las últimas décadas es, entre otros factores, responsable del aumento en la prevalencia de las especies de Candida no albicans. Algunas de ellas poseen resistencia natural a fluconazol (C. krusei) o elevada resistencia a este fármaco (C. glabrata). Se desconoce la prevalencia de estas especies a nivel nacional, situándose estimativamente entre 5 y $20 \%$ del total de aislados de Candida.

Finalmente, desde el punto de vista de los mecanismos de acción, fluconazol es un fármaco fungistático, lo que entre otros factores podría explicar hallazgos como los del estudio de Reboli y cols, en el que se comparó fluconazol con equinocandinas (anidulafungina) observándose una mayor eficacia clínica de este segundo, incluso en pacientes que presentaban C. albicans ${ }^{32}$.

En relación a los potenciales efectos adversos secundarios a su uso, fluconazol ha sido asociado a prolongación del intervalo QT y alteración en las pruebas hepáticas, lo que puede llegar a necesitar suspensión de éste. Debido a su metabolismo hepático a nivel de isoformas del citocromo $\mathrm{P}-450$, pueden presentarse interacciones droga-droga, entre otras con antidepresivos tricíclicos, zidovudina, rifampici- na, benzodiacepinas, ciclosporina y fenitoína, lo que puede determinar cambios en los niveles plasmáticos de estos fármacos ${ }^{33}$. Asimismo, dado cambios farmacocinéticos frecuentes en los pacientes críticos, en particular aquellos obesos, con sepsis y quemados, los niveles plasmáticos de fluconazol son muy variables ${ }^{34}$.

Tabla 3. Antifúngicos empleados en candidiasis invasora: Eficacia clínica, seguridad y costos directos

\begin{tabular}{llll} 
& Eficacia & Seguridad & Costo \\
Imidazólicos & & & \\
Fluconazol & Buena $^{1}$ & Adecuada & Bajo \\
Voriconazol & Muy buena & Adecuada $^{2}$ & Intermedio \\
Polienos & & & \\
Anfotericina B deoxicolato & Muy buena & $>$ tasa efectos adversos $^{3}$ & Intermedio \\
Anfotericina B liposomal & Muy buena & Adecuada & Elevado \\
Equinocandinas & & & \\
Caspofungina & Muy buena & Adecuada ${ }^{4}$ & Elevado \\
Anidulafungina & Muy buena & Adecuada & \\
Micafungina & Muy buena & Adecuada & Elevado \\
\hline
\end{tabular}

'Resistencia intrínseca a fluconazol en Candida krusei e intermedia (30-50\% de las cepas) para Candida glabrata. ${ }^{2}$ Dado el metabolismo hepático de voriconazol, existe riesgo de interacciones con diversos fármacos que comparten su metabolismo. ${ }^{3}$ Superiores a fluconazol y a caspofungina en ensayos clínicos, insuficiencia renal con necesidad de diálisis. ${ }^{4}$ Ensayo clínico versus anfotericina B. ${ }^{5}$ Ensayo clínico contra fluconazol.

\begin{tabular}{|c|c|c|}
\hline & Dosis & Duración \\
\hline \multicolumn{3}{|l|}{ Imidazólicos } \\
\hline Fluconazol & $\begin{array}{l}800 \text { mg i.v. inicial, } \\
400 \text { mg i.v. día, mantención }\end{array}$ & 14 días $^{1}$ \\
\hline \multirow[t]{2}{*}{ Voriconazol } & 6 mg/kg cada 12 horas día 1 & \\
\hline & 3 mg/kg cada 12 horas mantención & 14 días $^{1}$ \\
\hline \multicolumn{3}{|l|}{ Polienos } \\
\hline Anfotericina $B$ deoxicolato & 0,5 - 1,0 mg/kg/día & Dosis total $1-1,5 \mathrm{grs}$ \\
\hline Anfotericina B liposomal & $3-5 \mathrm{mg} / \mathrm{kg} / \mathrm{día}$ & Dosis total $1-1,5$ grs \\
\hline \multicolumn{3}{|l|}{ Equinocandinas } \\
\hline Caspofungina & $\begin{array}{l}70 \text { mg i.v. dosis de carga } \\
50 \text { mg i.v. día, mantención }\end{array}$ & 14 días $^{1}$ \\
\hline Anidulafungina & $\begin{array}{l}200 \text { mg i.v. dosis de carga } \\
100 \text { mg i.v. día, mantención }\end{array}$ & 14 días $^{1}$ \\
\hline Micafungina & 100 mg i.v. día & 14 días $^{1}$ \\
\hline \multicolumn{3}{|c|}{$\begin{array}{l}\text { 'Los ensayos clínicos de los diversos antifúngicos en candidemia han empleado } 14 \text { días desde el úl- } \\
\text { timo hemocultivo positivo como tiempo mínimo de tratamiento. Las Guías de la IDSA recomiendan } \\
\text { completar al menos } 14 \text { días de terapia, en la presencia de evolución clínica favorable, y ausencia de } \\
\text { complicación a distancia. }\end{array}$} \\
\hline
\end{tabular}


Voriconazol. Es un triazol de segunda generación, disponible en el mercado chileno, cuya principal indicación es la terapia de infecciones fúngicas por hongos filamentosos. Posee cobertura adecuada para infecciones por Candida spp, contando con aprobación de FDA y EMEA para el tratamiento de la candidemia en pacientes no neutropénicos. Como fluconazol, voriconazol es un fármaco fungistático de metabolismo hepático, por lo cual puede interactuar con otros medicamentos que se metabolizan a ese nivel. Además, su uso tiene algunas complicaciones logísticas en esta población de pacientes: no se recomienda administrar el fármaco por sonda enteral y existe contraindicación relativa para el empleo de la formulación endovenosa en pacientes con insuficiencia renal, condición frecuente en UCI. En general, voriconazol es bien tolerado, pero se le ha asociado a efectos adversos visuales, anormalidad en las pruebas hepáticas y rash cutáneo.

Por lo mencionado, y pese a tener una adecuada cobertura para esta indicación, voriconazol se emplea infrecuentemente para la terapia de CI.

Posaconazol y ravuconazol. Son nuevos antifúngicos pertenecientes a la familia de los triazoles. Poseen adecuada actividad in vitro e in vivo contra especies de Candida. El uso de posaconazol está documentado en la profilaxis de infecciones fúngicas en pacientes con neutropenia. Posaconazol está recomendado además en candidiasis invasora refractaria a otros antifúngicos. No existen a la fecha ensayos clínicos que apoyen el empleo de estos fármacos en la CI en no inmunosuprimidos. Recientemente han sido interrumpidos los estudios clínicos con ravuconazol.

Anfotericina $\boldsymbol{B}$ deoxicolato. Es el fármaco más tradicional empleado con esta indicación. Posee una adecuada actividad fungicida contra las diversas especies de Candida; sin embargo, su uso se asocia a frecuentes y, en ocasiones, graves efectos adversos. Fiebre, exantema, hipokalemia e insuficiencia renal se encuentran dentro de las más relevantes ${ }^{35}$. El desarrollo de insuficiencia renal es un hecho que alcanza a $10-40 \%$ de los pacientes adultos, según sea la definición empleada. La incidencia de insuficiencia renal fue de casi $25 \%$ en el estudio de Mora-Duarte y cols que comparó anfotericina-B con caspofungina, muy superior al $8,4 \%$ observado en el grupo que recibió equinocandinas ${ }^{36}$. El desarrollo de novo de insuficiencia renal en un paciente crítico ha sido asociado a un incremento en la mortalidad y los costos.

Anfotericina B liposomal. Corresponde a una preparación de anfotericina desarrollada a fines de la década de los 80 `en base a una emulsión lipídica que limita el contacto de anfotericina $\mathrm{B}$ con las membranas biológicas. Mediante este mecanismo se reducen los efectos adversos de ella, sin cambiar la eficacia clínica. Ello permite además el empleo de dosis mayores de anfotericina $\mathrm{B}$.
Evaluada inicialmente en uso compasivo para la terapia de pacientes neutropénicos febriles refractarios a otros antifúngicos, posteriormente se estudió en otras infecciones fúngicas ${ }^{37,38}$. Protocolos más recientes en que se comparó directamente con equinocandinas (micafungina), documentan similar eficacia a equinocandinas, pero se observó una mayor tasa de eventos adversos relacionados al fármaco en el grupo anfotericina liposomal ${ }^{39}$. En virtud de su elevado costo y de la presencia de otras altenativas para el tratamiento de la candidemia en pacientes críticos adultos, su uso en esta indicación es inusual.

Equinocandinas. Constituyen una familia de antifúngicos fungicidas desarrollada a partir de la década de los 90'. Los exponentes actuales de la familia son caspofungina, anidulafungina y micafungina, quienes comparten el mecanismo de acción, cual es inhibir la acción de la 1,3-b-D glucano sintetasa. De esta forma se inhibe la generación de la pared celular tanto de Candida spp, como de otras especies de hongos. Este mecanismo explica también la baja tasa de incidencia de efectos adversos, al no existir esta enzima en las células humanas. Representan un grupo de fármacos que inhibe la proliferación del patógeno, sin afectar las células del individuo que desarrolla la enfermedad.

Para todos los exponentes de la familia, se cuenta con adecuada evidencia en relación a su eficacia y seguridad $^{32,36,39}$. Poseen diferencias farmacodinámicas y farmacocinéticas que determinan la dosificación y el potencial de interacción con otros fármacos, así como la necesidad de ajuste de dosis en presencia de disfunción renal y/o hepática.

Los estudios más relevantes que validan el rol de las equinocandinas en los pacientes con candidemia son los de Mora-Duarte, Reboli, y Kuse previamente comentado. El primero de ellos comparó caspofungina con anfotericinaB deoxicolato en 239 pacientes con candidemia o aislado de cándida a partir de un sitio estéril. El desenlace primario- eficacia global al fin de la terapia endovenosa- fue similar en ambos grupos, $73,4 \%$ caspofungina y $61,7 \%$ anfotericina-B. Como se mencionó previamente, la incidencia de efectos adversos fue superior en los pacientes que recibieron anfotericina-B deoxicolato $(58,4 \%$ versus $28,9 \%$ en el grupo caspofungina). Caspofungina redujo significativamente la presencia de calofríos, fiebre, alteraciones de laboratorio, eventos relacionados a la infusión, hipokalemia y nefrotoxicidad.

El estudio de Reboli comparó anidulafungina con fluconazol en 245 pacientes. Su desenlace primario fue la tasa de éxito global al finalizar la terapia endovenosa. Pese a ser un estudio de no inferioridad, se documentó una mayor eficacia clínica de anidulafungina $(75,6 \%)$ que fluconazol $(60,2 \%)$, (p 0,01). No hubo diferencia en la tasa de efectos adversos globales en ambos grupos; no 
obstante, más pacientes del grupo fluconazol debieron ser retirados del estudio por efectos adversos.

La aparición de este estudio ha fortalecido la evidencia disponible respecto al empleo de equinocandinas ante esta indicación, ya que fue el primero que documentó mayor eficacia clínica, motivando provocadoras editoriales en relación a su empleo en pacientes con infecciones por cándida ${ }^{40}$.

A la fecha, sólo un estudio ha comparado directamente dos equinocandinas, sin encontrarse diferencias significativas entre caspofungina y micafungina ${ }^{41}$.

Lamentablemente, las equinocandinas mantienen un elevado costo de adquisición, lo que ha limitado su empleo en nuestro sistema público de salud. Sin embargo, esto debiera mejorar ya que la introducción de nuevos miembros a la familia de equinocandinas debería conducir a una reducción en los precios, haciéndolos más accesibles a todos los centros hospitalarios.

Otro aspecto clave para lograr una mejoría en los desenlaces de pacientes con CI es el impacto del tiempo de inicio de la terapia antifúngica. De forma similar al comportamiento de la antibioticoterapia en pacientes con sepsis grave, estudios retrospectivos han documentado el impacto en mejoría de desenlaces del inicio precoz de antifúngicos ${ }^{42,43}$. Esta información apoya fuertemente la práctica del inicio de terapia antifúngica adecuada dentro de las primeras 24 horas de la sospecha clínica de CI, como un factor que mejora la evolución de estos pacientes. Se estima que una ventana entre la aparición de la candidemia y el inicio de la terapia de más de 24 hrs., podría aumentar las tasas de mortalidad hasta en $20 \%$ absoluto.

En virtud de los antecedentes expuestos, el inicio de terapia antifúngica precoz y eficaz en pacientes con sospecha clínica de CI es clave para intentar reducir su morbilidad y mortalidad. Esta precocidad, sin embargo, debe balancearse con el uso juicioso de antifúngicos, de manera de limitar su uso indiscriminado, sus potenciales consecuencias económicas y el desarrollo de resistencia.

Estos aspectos han sido recientemente analizados de manera extensa en una revisión europea acerca del tema y bajo el prisma de la medicina basada en la evidencia, en la revisión 2009 de las Guías IDSA (Infectious Diseases Society of America) $)^{1,44}$. Estas guías constituyen un relevante documento que analizaremos a continuación, con énfasis en las recomendaciones correspondientes al empleo de antifúngicos ante la sospecha o documentación de candidemia o CI en pacientes no neutropénicos.

- Para los pacientes no neutropénicos, el esquema sugerido es similar en las infecciones sospechadas o documentadas. En ambos escenarios se sugiere el empleo de fluconazol, caspofungina, micafungina o anidulafungina como terapia inicial.

- Se recomienda el empleo de equinocandinas en pa- cientes con exposición reciente a azoles, pacientes en riesgo elevado de C. glabrata o C. krusei y en aquellos que se manifiestan como una enfermedad moderada a grave.

- Se limita el empleo de anfotericina-B a aquellos pacientes intolerantes a otros antifúngicos y en casos de limitaciones en el acceso a equinocandinas.

- La recomendación cada vez más fuerte del empleo de equinocandinas en pacientes que desarrollen CI es también el mensaje global de la reciente revisión del grupo europeo de estudio de infecciones fúngicas.

En nuestra opinión, esta recomendación está más que razonablemente documentada, en virtud de la eficacia y seguridad que han evidenciado los miembros de esta familia. Sin embargo, más allá de la recomendación efectuada, queda una serie de aspectos no resueltos en esta condición, que requieren de más investigación.

En primer lugar, es necesario conocer la epidemiología institucional. Si un centro aún mantiene una proporción inferior a $5-10 \%$ de C. glabrata y C. krusei, podría ser razonable privilegiar el uso empírico de fluconazol.

Un segundo conflicto- frecuente en pacientes críticos en que se sospecha CI y se decide inicio empírico de antifúngicos- es que los resultados de los cultivos sean negativos. Qué hacer con la indicación de antifúngicos en presencia de cultivos sin desarrollo de Candida es, a la fecha, una decisión del equipo tratante, que depende del status clínico, la documentación o no de algún diagnóstico diferencial capaz de explicar el deterioro clínico y la evolución clínica durante los días de terapia. En nuestra opinión, en presencia de cultivos negativos y documentación fehaciente de otro diagnóstico diferencial, debería considerarse la suspensión del antifúngico.

Finalmente, otra situación frecuente y en la cual sí existe un comentario en las recientes recomendaciones, es aquella en que se documenta un cultivo positivo a Candida sp sensible a fluconazol y el paciente inició equinocandinas empíricamente. En estos casos se recomienda ajustar el esquema a fluconazol, siempre que las condiciones clínicas del paciente lo posibiliten.

Debiera sospecharse la presencia de un brote nosocomial de infección fúngica invasora ante un incremento en la incidencia de infecciones por cándida, causados por una misma especie. En conjunto con evaluar los procesos de control de infección intrahospitalaria (IIH), debiera plantearse la realización de estudios microbiológicos complementarios para evaluar la clonalidad de los aislados de Candida sp.

\section{Conclusión}

El diagnóstico y apropiado tratamiento de la CI se mantienen como un tremendo desafío, tanto para el 
especialista en cuidados intensivos como para el infectólogo. Una adecuada interacción entre ambos equipos y una apropiada ponderación de la presencia de factores de riesgo, colonización por Candida spp y gravedad del cuadro clínico que motiva la sospecha clínica, favorecen un diagnóstico certero de esta condición. La eficacia y seguridad de las equinocandinas las ubica como los fármacos de elección para el manejo del paciente con sospecha de CI hospitalizado en la unidad de paciente crítico. No obstante, es necesario un uso juicioso de este grupo de medicamentos, de manera de limitar la aparición de resistencia antifúngica y evitar un consumo de recursos excesivos.

\section{Resumen}

Las infecciones invasoras por Candida spp, represen- tan una patología relevante en los pacientes críticos. Para su oportuno diagnóstico es necesaria una elevada sospecha clínica, tomando en consideración el cuadro clínico y la presencia de factores de riesgo. Pese a la incorporación de nuevos fármacos al arsenal terapéutico durante la última década, mantiene una elevada mortalidad. Las claves para mejorar los desenlaces clínicos en estos pacientes son el empleo de una terapia precoz, eficaz y que permita la cobertura de distintas especies de Candida: C albicans y no albicans. Recientes guías internacionales sugieren la terapia empírica con equinocandinas ante la sospecha de candidiasis invasora en esta población de pacientes. Este grupo de fármacos ha documentado adecuada eficacia clínica y seguridad en estos pacientes. Se espera que la incorporación de nuevas equinocandinas al mercado aminore sus costos y mejore el acceso a este grupo de fármacos.

\section{Referencias}

1.- Pappas P G, Kauffman C A, Andes D, Benjamin D K Jr, Calandra TF, Edwards J E $\mathrm{Jr}$, et al. Clinical practice guidelines for the management of candidiasis: 2009 update by the Infectious Diseases Society of America. Clin Infect Dis 2009; 48: 503-35.

2.- Almirante B, Rodríguez D, Park B J, CuencaEstrella M, Planes A M, Almela M, et al. Epidemiology and predictors of mortality in cases of Candida bloodstream infection: results from population-based surveillance, barcelona, Spain, from 2002 to 2003. J Clin Microbiol 2005; 43: 1829-35.

3.- Wisplinghoff H, Bischoff T, Tallent SM, Seifert H, Wenzel RP, Edmond MB. Nosocomial bloodstream infections in US hospitals: analysis of 24,179 cases from a prospective nationwide surveillance study. Clin Infect Dis 2004; 39 : 309-17.

4.- Colombo A L, Nucci M, Park B J, Nouer S A, Arthington-Skaggs B, da Matta D A, et al. Epidemiology of candidemia in Brazil: a nationwide sentinel surveillance of candidemia in eleven medical centers. J Clin Microbiol 2006; 44: 2816-23.

4a.- Nucci M, Queiroz-Telles F, Tobón A M, Restrepo A, Colombo A L. Epidemiology of opportunistic fungal infections in Latin America. Clin Infect Dis 2010; 51 (5): 561-70.

5.- Morgan J, Meltzer M I, Plikaytis B D, Sofair A N, Huie-White S, Wilcox S, et al. Excess mortality, hospital stay, and cost due to candidemia: a case-control study using data from population-based candidemia surveillance. Infect Control Hosp Epidemiol 2005; 26: 540-7.

6.- Gudlaugsson O, Gillespie S, Lee K, Vande Berg J, Hu J, Messer S, et al. Attributable mortality of nosocomial candidemia, revisited. Clin Infect Dis 2003; 37: 1172-7.

7.- Rentz A M, Halpern M T, Bowden R. The impact of candidemia on length of hospital stay, outcome, and overall cost of illness. Clin Infect Dis 1998; 27: 781-8.

8.- Falagas M E, Apostolou K E, Pappas V D. Attributable mortality of candidemia: a systematic review of matched cohort and casecontrol studies. Eur J Clin Microbiol Infect Dis 2006; 25: 419-25.

9.- Silva V, Díaz M C, Febre N. Invasive fungal infections in Chile: a multicenter study of fungal prevalence and susceptibility during a 1-year period. Med Mycol 2004; 42: 333-9.

10.- Horn D L, Neofytos D, Anaissie E J, Fishman J A, Steinbach W J, Olyaei A J, et al. Epidemiology and outcomes of candidemia in 2019 patients: data from the prospective antifungal therapy alliance registry. Clin Infect Dis 2009; 48: 1695-703.

11.- Marchetti O, Bille J, Fluckiger U, Eggimann P, Ruef C, Garbino J, et al. Epidemiology of candidemia in Swiss tertiary care hospitals: Secular trends, 1991-2000. Clin Infect Dis 2004; 38: 311-20.

12.- Tortorano A M, Biraghi E, Astolfi A, Ossi C, Tejada M, Farina C, and FIMUA Candidemia Study Group. European Confederation of Medical Mycology (ECMM). Prospective survey of candidemia: report from one Italian region. J Hosp Infect; 51: 297-304.

13.- Bassetti M, Righi E, Costa A, Fasce R, Molinari $\mathrm{M}$, Rosso R, et al. Epidemiological trends in nosocomial candidemia in intensive care. BMC Infectious Dis 2006;6: 21-6.

14.- Kung H C, Wang J L, Chang S C, Wang J T, Sun H Y, Hsueh P R, et al. Community-onset candidemia at a university hospital, 1995-2005.
J Microbiol Immunol Infect 2007; 40: 355-63.

15.- Shorr A F, Gupta V, Sun X, Johannes R S, Spalding J, Tabak Y P. Burden of early-onset candidemia: analysis of culture-positive bloodstream infections from a large U.S. database. Crit Care Med 2009; 37: 2519-26; quiz 2535.

16.- Khatib R, Thirumoorthi M C, Riederer K M, Sturm L, Oney L A, Baran J Jr. Clustering of candida infections in the neonatal intensive care unit: concurrent emergence of multiple Straits simulating intermittent outbreaks. Pediatr Infect Dis J 1998; 17 (2): 130-4.

17.- Dizbay M, Kalkanci A, Sezer B E, Aktas F, Aydogan S, Fidan I, Kustimur S, Sugita T. Molecular investigation of a fungemia outbreak due to Candida parapsilosis in an intensive care unit. Braz J Infect Dis 2008; 12 (5): 395-9.

18.- Pappas P G. Invasive candidiasis. Infect Dis Clin North Am 2006, 20 (3): 485-506

19.- Hardley S, Lee W W, Ruthazer R, Nasraway S A Jr. Candidemia as a cause of septic shock and multiple organic failure in noninmunocompromised patients. Crit Care Med 2002; 30(8): 1808-14.

20.- Guery B P, Arendrup M C, Auzinger G, Azoulay E, Borges Sá M, Johnson E M, et al. Management of invasive candidiasis and candidemia in adult non-neutropenic intensive care unit patients: Part I. Epidemiology and diagnosis. Intensive Care Med 2009; 35: 55-62.

21.- O'Grady N P, Barie P S, Bartlett J G, Bleck T, Carroll K, Kalil A C, et al. Guidelines for evaluation of new fever in critically ill adult patients: 2008 update from the American Collegue of Critical Care Medicine and the Infectious Disease Society of America. Crit Care Med 2008; 36(4): 1330-49. 
22.- Voss A, Hollis R J, Pfaller M A, Wenzel R P, Doebbeling B N. Investigation of the sequence of colonization and candidemia in nonneutropenic patients. J Clin Microbiol 1994; 32: 975-80.

23.- Pittet D, Monod M, Suter P M, Frenk E, Auckenthaler R. Candida colonization and subsequent infections in critically ill surgical patients. Ann Surg 1994; 220: 751-8.

24.- Leon C, Ruiz-Santana S, Saavedra P, Almirante B, Nolla-Salas J, Álvarez-Lerma F, et al. A bedside scoring system ("Candida score") for early antifungal treatment in nonneutropenic critically ill patients with candida colonization. Crit Care Med 2006; 34: 730-7.

25.- León C, Ruiz-Santana S, Saavedra P, Galvan B, Blanco A, Castro C, et al. Usefulness of the "Candida score" for discriminating between candida colonization and invasive candidiasis in non-neutropenic critically ill patients: a prospective multicenter study. Crit Care Med 2009; 37: 1624-33.

26.- Bennett J. Is real-time polymerase chain reactions ready for real use in detecting candidemia? Clin Infect Dis 2008; 46: 897-8.

27.- McMullan R, Metwally L, Coyle P V, Hedderwick S, McCloskey B, O'Neill H J, et al. A prospective clinical trial of a realtime polymerase chain reaction assay for the diagnosis of candidemia in nonneutropenic, critically ill adults. Clin Infect Dis 2008; 46: 890-6.

28.- Kedzierska A, Kochan P, Pietrzky A, Kedzierska J. Current status of fungal wall components in the inmunodiagnostics of invasive fungal infections in humans: galactomannan, mannan and (1 -->3)-beta-Dglucan antigens. Eur J Clin Microbiol Infect Dis 2007; 26 (11): 755-66.

29.- Lass-Flörl C, Perkhofer V, Mayr A. In vitro susceptibility testing in fungi: a global perspective on a variety of methods. Mycoses
2010; 53 (1): 1-11

30.- Garbino J, Lew D P, Romand J A, Hugonnet S, Auckenthaler R, Pittet D. Prevention of severe Candida infections in nonneutropenic, high-risk, critically ill patients: a randomized, doubleblind, placebo-controlled trial in patients treated by selective digestive decontamination. Intensive Care Med 2002; 28: 1708-17.

31.- Piarroux R, Grenouillet F, Balvay P, Tran V, Blasco G, Millon L, et al. Assessment of preemptive treatment to prevent severe candidiasis in critically ill surgical patients. Crit Care Med 2004; 32: 2443-9.

32.- Reboli A C, Rotstein C, Pappas P G, Chapman S W, Kett D H, Kumar D, et al. Anidulafungin versus fluconazole for invasive candidiasis. N Engl J Med 2007; 356: 2472-82.

33.- Gupta A K, Katz H I, Shear N H. Drug interactions with itraconazole, fluconazole, and terbinafine and their management. J Am Acad Dermatol 1999; 41 (2): 237-49

34.- Pittrow L, Penk A. Special pharmacokinetics of fluconazole in septic, obese and burn patients. Mycosis 1999; 42 Suppl 2: 87-90.

35.- Washington C, Lance M, Davis S S. Toxicity of amphotericin $\mathrm{B}$ emulsion formulations. J Antimicrob Chemother 1993; 31: 806-8.

36.- Mora-Duarte J, Betts R, Rotstein C, Colombo A L, Thompson-Moya L, Smietana J, et al. Comparison of caspofungin and amphotericin B for invasive candidiasis. N Engl J Med 2002; 347: 2020-9.

37.- Kline S, Larsen TA, Fieber L, Fishbach R, Greenwood M, Harris R, et al. Limited toxicity of prolonged therapy with high doses of amphotericin B lipid complex. Clin Infect Dis 1995; 21: 1154-8.

38.- Heinemann V, Bosse D, Jehn U, Kähny B, Wachholz K, Debus A, et al. Pharmacokinetics of liposomal amphotericin B (Ambisome) in critically ill patients. Antimicrb Agents Chemother 1997; 41 (6): 1275-80.

39.- Kuse E R, Chetchotisakd P, da Cunha C A,
Ruhnke M, Barrios C, Raghunadharao D, et al. Micafungin versus liposomal amphotericin $\mathrm{B}$ for candidaemia and invasive candidosis: a phase III randomised double-blind trial. Lancet 2007; 369: 1519-1527.

40.- Sobel J D, Revankar S G. Echinocandins: first-choice or first-line therapy for invasive candidiasis ? N Engl J Med 2007; 356 (24): 2525-6.

41.- Pappas P G, Rotstein C M, Betts R F, Nucci M, Talwar D, De Waele J J, et al. Micafungin versus caspofungin for treatment of candidemia and other forms of invasive candidiasis. Clin Infect Dis 2007; 45: 883-93.

42.- Garey K W, Rege M, Pai M P, Mingo D E, Suda K J, Turpin R S, et al. Time to initiation of fluconazole therapy impacts mortality in patients with candidemia: a multi-institutional study. Clin Infect Dis 2006; 43: 25-31.

43.- Patel G P, Simon D, Scheetz M, Crank C W, Lodise T, Patel N. The effect of time to antifungal therapy on mortality in Candidemia associated septic shock. Am J Ther 2009; 16 (6): 508-11.

44.- Guery B P, Arendrup M C, Auzinger G, Azoulay E, Borges Sá M, Johnson E M, et al. Management of invasive candidiasis and candidemia in adult non-neutropenic intensive care unit patients: Part II. Treatment. Intensive Care Med 2009; 35: 206-14.

45.- Fraser V J, Jones M, Dunkel J, Storfer S, Medoff G, Dunagan W C. Candidemia in a tertiary care hospital: epidemiology, risk factors, and predictors of mortality. Clin Infect Dis 1992; 15: 414-21.

46.- Morgan J. Global trends in candidemia: review of reports from 1995-2005. Curr Infect Dis Rep 2005; 7: 429-39.

47.- Chow J K, Golan Y, Ruthazer R, Karchmer A W, Carmeli Y, Lichtenberg DA, et al. Risk factors for albicans and non-albicans candidemia in the intensive care unit. Crit Care Med 2008; 36: 1993-8. 